\title{
REVISÃo de Literatura Dependência de internet e transtorno de déficit de atenção com hiperatividade (TDAH): revisão integrativa
}

\author{
Internet Addiction and Attention Deficit Hyperactivity \\ Disorder (ADHD): integrative review of the literature
}

Helena Cristina Medeiros Vieira Schmidek1, Julia Corrêa Gomes², Patricia Leila dos Santos³, Ana Maria Pimenta de Carvalho ${ }^{4}$, Luiz Jorge Pedrão $0^{4}$, Clarissa Mendonça Corradi-Webster ${ }^{5}$

\section{RESUMO}

Objetivo: Realizar uma revisão integrativa identificando na literatura trabalhos que pudessem sintetizar resultados que refletissem a possível relação entre dependência de internet (DI) e transtorno de déficit de atenção com hiperatividade (TDAH) na população adolescente, bem como achados desses estudos que agreguem informações para o aprimoramento e diagnóstico de DI. Métodos: Revisão integrativa da literatura em três bases de dados: PsycINFO, Scopus e Cinahal, com os seguintes termos: "internet addiction", "adolescent" e "attention deficit disorder with hyperactivity", de 2007 a 2017, com informações sobre a relação da dependência de internet e o TDAH. Resultados: A amostra ficou composta por 12 artigos originais, provenientes, em sua maioria, da Ásia e Oriente Médio. De sua análise emergiram as seguintes categorias: Relação entre DI e TDAH; Outros fatores associados à DI; Instrumentos para avaliação de DI e dados de incidência. Há ausência de critérios definidos de diagnóstico de dependência de internet, tendo sido identificados quatro instrumentos para mensurar a $\mathrm{Dl}$, todos os questionários foram preenchidos pelos próprios adolescentes, com índices na população variando entre $2,4 \%$ e 10,6\%. Nos adolescentes diagnosticados com DI, as desor-

\section{Palavras-chave}

Internet, transtorno de déficit de atenção com hiperatividade, adolescente, comportamento aditivo, dependência de internet. dens de ansiedade e depressão foram as comorbidades mais citadas depois do TDAH. Conclusões: Há evidências de associação importante entre os dois transtornos identificados, sem resultados conclusivos quanto ao processo da interação. Torna-se necessário o avanço em pesquisas visando a uma definição de critérios diagnósticos da DI, taxas de prevalência e preditores do problema, de modo a poder estabelecer intervenções terapêuticas e estratégias de prevenção do problema.

1 Universidade de São Paulo, Escola de Enfermagem de Ribeirão Preto (USP-EERP), Ribeirão Preto, SP, Brasil. 2 Universidade de São Paulo, Faculdade de Filosofia, Ciências e Letras de Ribeirão Preto (USP-FFCLRP), Ribeirão Preto, SP, Brasil.

3 Universidade de São Paulo, Faculdade de Medicina de Ribeirão Preto (USP-FMRP), Departamento de Neurociências e Ciências do Comportamento, Ribeirão Preto, SP, Brasil.

Recebido em

$5 / 9 / 2017$

Aprovado em

$25 / 1 / 2018$
4 Universidade de São Paulo, Escola de Enfermagem de Ribeirão Preto (USP-EERP), Departamento de Enfermagem Psiquiátrica e Ciências Humanas, Ribeirão Preto, SP, Brasil.

5 Universidade de São Paulo, Faculdade de Filosofia, Ciências e Letras de Ribeirão Preto (USP-FFCLRP), Departamento de Psicologia, Ribeirão Preto, SP, Brasil. 


\section{Keywords}

Internet, attention deficit hyperactivity disorder, adolescent, behavior addictive, internet addiction.

\section{ABSTRACT}

Objective: To perform an integrative review of the possible relationship between internet addiction (ID) and attention deficit hyperactivity disorder (ADHD) in adolescents aged 12 to 18 years. Also to gather informations in these studies for a more efficient diagnosis of DI. Methods: Integrative review of the literature in three databases: PsycINFO, Scopus and Cinahal, with the following terms: "internet addiction", "adolescent" and "attention deficit disorder with hyperactivity", 2007-2017, with information about the relationship between Internet addiction and ADHD. Results: The sample consisted of 12 original articles, mostly from Asia and the Middle East. From their analysis, the following categories emerged: Relationship between ID and ADHD; Other factors associated with ID; Instruments for evaluation of ID and incidence data. There is a lack of defined criteria for diagnosis of internet addiction, and four instruments were identified to measure ID, all questionnaires were completed by the adolescents themselves, with rates ranging from 2.4\% to 10.6\%. Anxiety and depression disorders were the most cited comorbidities after ADHD in the adolescents diagnosed with ID. Conclusions: There are evidences of an important association between the two identified disorders, however without conclusive results on the interaction process. In order to establish therapeutic interventions and strategies to prevent the problem, it is necessary to advance research on the definition of the diagnostic criteria of ID, prevalence rates and predictors of the problem.

\section{INTRODUÇÃO}

O desenvolvimento e o uso da tecnologia cada vez mais se expandem e ocupam espaços de atividades diárias, fazendo com que o computador seja veículo para diversas possibilidades de expressões e tarefas. De modo repentino, nas últimas décadas, a internet revolucionou a maneira como as pessoas aprendem, trabalham e, principalmente, interagem umas com as outras. Apesar dos inúmeros benefícios e facilidades proporcionados, essa ferramenta também pode ocasionar danos aos indivíduos que a utilizam demasiadamente. Existem diversas terminologias utilizadas para descrever problemas relacionados ao uso excessivo da internet? Será utilizado nesta revisão o termo "internet addiction" (IA), traduzido para o português (a partir de diferentes leituras) como "dependência de internet" (DI). Esse termo foi descrito por Young em 1996, para designar uma série de comportamentos das pessoas que podem desenvolver desconfortos emocionais associados a dificuldades em controlar o seu uso da internet, acarretando com isso prejuízos tanto no âmbito profissional quanto familiar ou social, de jovens e adultos, devido ao acesso em demasia. O diagnóstico de DI é difícil e complicado, já que a internet é uma ferramenta tecnológica muito utilizada e pelo fato de que, atualmente, não existe um conjunto aceito de critérios para a dependência listados no Manual Diagnóstico e Estatístico de Transtornos Mentais². Apesar da falta de consenso entre os pesquisadores a respeito dos critérios diagnósticos da DI, muitos apontam para uma similaridade entre esse comportamento e o transtorno por uso de substâncias, tanto no funcionamento comportamental quanto no neurobiológico’.
No Brasil, o Estatuto da Criança e do Adolescente (ECA), Lei no 8.069, de 1990, define a adolescência como um período da vida compreendido entre a faixa etária dos 12 aos 18 anos de idade 3 . A adolescência é uma fase crítica para o desenvolvimento de comportamentos de risco e, devido ao crescente tempo gasto pelos jovens utilizando a internet, faz-se importante estudar essa população em sua relação com o uso excessivo dessa tecnologia4. É também uma fase caracterizada pela imaturidade dos sistemas cerebrais monoaminérgicos cortical frontal e subcortical, o que faz com que a impulsividade seja um traço comportamental transitório típico dessa etapa ${ }^{5}$. Esse dado pode explicar o fato de os adolescentes possuírem menos habilidade em controlar o entusiasmo por algo que lhes desperta interess $\mathrm{e}^{6}$ e serem os principais acometidos pela $\mathrm{Dl}^{7}$.

Estudos têm apontado para uma alta comorbidade de DI com outros transtornos mentais, destacando-se em incidência o transtorno de déficit de atenção com hiperatividade (TDAH) e outros transtornos psiquiátricos ${ }^{6,8}$.

OTDAH é um transtorno do desenvolvimento de forte influência neurobiológica, com etiologia multifatorial, incluindo fatores genéticos e ambientais. Sua prevalência mundial é de cerca de 5,29\% em crianças e adolescentes ${ }^{9}$, o que determina uma grande procura por atendimento médico e psicopedagógico ${ }^{10}$. É caracterizado por desatenção, tendência à distração, impulsividade e excessiva atividade motora em graus inadequados à sua etapa do desenvolvimento. A princípio, a hiperatividade e a reduzida capacidade de concentração nos indivíduos com TDAH poderiam sugerir dificuldade em permanecer na internet, e consequentemente 
uma não associação com a D|8,11. Há, no entanto, indícios de que o TDAH pode influenciar o surgimento da DI ${ }^{11}$.

Diante desse contexto, levantou-se a seguinte questão: o que a comunidade científica que se dedica à investigação da DI já conhece sobre a possível relação entre DI e TDAH em indivíduos de 12 a 18 anos de idade? Assim, o objetivo desta investigação foi realizar uma revisão integrativa no período entre 2007 e 2017, identificando na literatura trabalhos que pudessem sintetizar resultados que refletissem a possível relação entre a DI e o TDAH na população adolescente, bem como achados desses estudos que agreguem informações para o aprimoramento e o diagnóstico de DI.

\section{MÉTODOS}

O levantamento bibliográfico foi conduzido concomitantemente pelas duas primeiras autoras, buscando-se minimizar possíveis vieses no processo de identificação e seleção dos documentos. Foram consultadas três bases de dados: PsycINFO, Scopus e Cinahal. Considerando a dificuldade em identificar descritores precisos para o termo "internet addiction", optou-se pelos seguintes termos, por resultarem em um número maior de documentos afins à temática investigada: "internet addiction", "adolescent" e "attention deficit disorder with hyperactivity". Foi utilizado, em todas as bases de dados, o operador booleano AND conectando os termos selecionados e foi considerado apenas um limite de busca: artigos indexados no período compreendido entre os anos 2007 e 2017.
A leitura primária de títulos e resumos foi realizada conjuntamente, sendo então aplicados, critérios adicionais de inclusão e exclusão com vistas a selecionar apenas aqueles artigos que potencialmente respondessem ao objetivo da presente revisão.

Os critérios de inclusão foram: estudos sobre DI que buscavam conhecer a relação entre DI e TDAH, realizados com adolescentes de 12 a 18 anos; artigos publicados em espanhol, português ou inglês. Os critérios de exclusão foram: estudos em não humanos; estudos relacionados com bullying virtual; estudos que investigassem o uso de jogos eletrônicos e não distinguissem entre jogos on-line e off-line; estudos com foco apenas no diagnóstico ou no tratamento do TDAH ou da Dl; artigos repetidos.

Após a leitura completa dos textos selecionados, dúvidas e divergências quanto a inclusão/exclusão de artigos foram discutidas conjuntamente pelos pesquisadores e decididas por consenso. O processo de seleção dos artigos está sumarizado na Figura 1.

\section{RESULTADOS}

A amostra ficou então composta por 12 artigos originais, todos escritos na língua inglesa, sobre a relação entre DI e TDAH em adolescentes. Foi organizada uma planilha para extração dos dados, com as características dos estudos e seus principais achados, que é apresentada na Tabela 1.

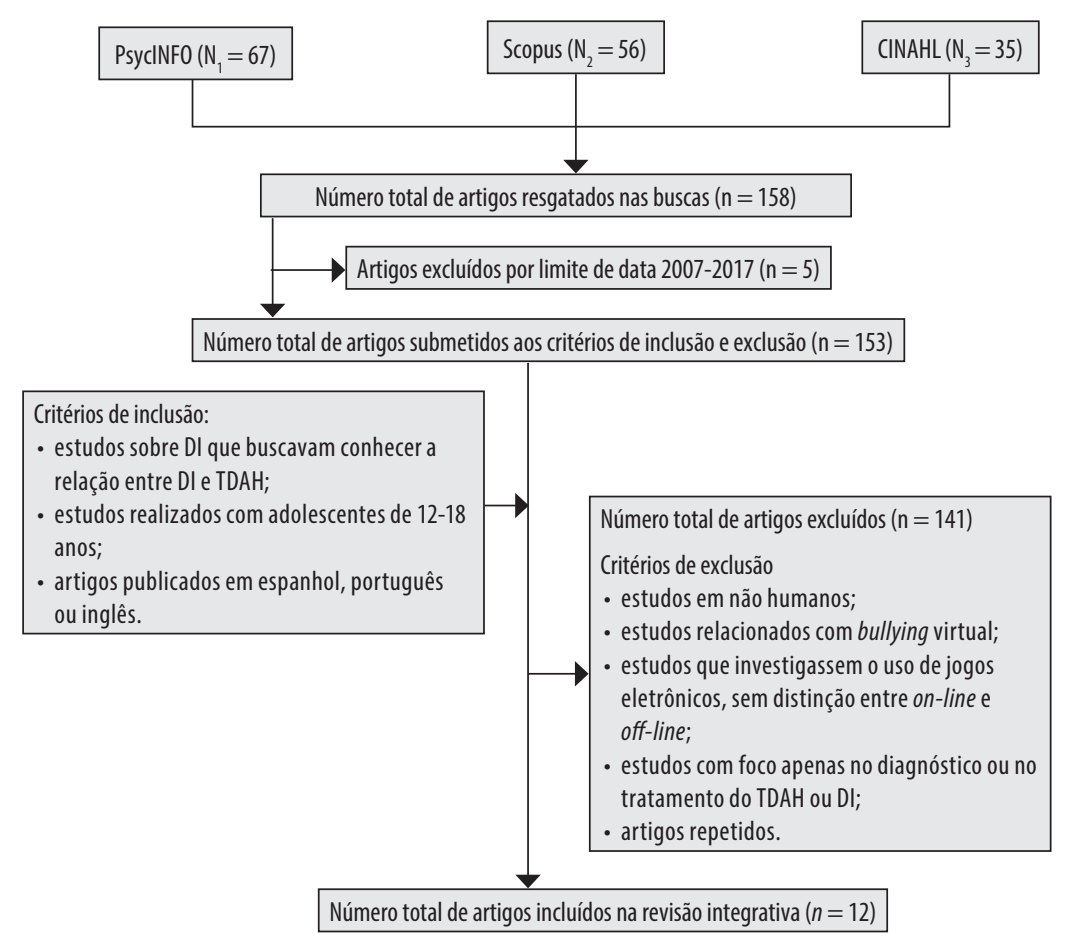

Figura 1. Fluxo de seleção de artigos que investigam a relação entre DI e TADH. 
Tabela 1. Descrição dos artigos selecionados em ordem crescente do ano de publicação

\begin{tabular}{|c|c|c|c|c|c|c|}
\hline Referência completa & País & $\begin{array}{c}\text { Desenho } \\
\text { de pesquisa }\end{array}$ & Objetivo & Amostra & Instrumentos & Principais resultados \\
\hline $\begin{array}{l}\text { Cao F, Su L, Liu T, Gao X. The } \\
\text { relationship between impulsivity } \\
\text { and Internet addiction in a sample of } \\
\text { Chinese adolescents. Eur Psychiatry. } \\
\text { 2007;22(7):466-71.12 }\end{array}$ & China & $\begin{array}{l}\text { Estudo } \\
\text { transversal }\end{array}$ & $\begin{array}{l}\text { Obter informações sobre } \\
\text { a relação entre a DI e } \\
\text { a impulsividade entre } \\
\text { adolescentes chineses. }\end{array}$ & $\begin{array}{c}\mathrm{n}=2.620 \\
12-18 \text { anos } \\
\text { Masc.: } 1.316 \\
\text { Fem.: } 1.304\end{array}$ & $\begin{array}{l}\text { - YDQ; } \\
\text { - DSM-IV; } \\
\text { - K-SADS; } \\
\text { - BIS-11; } \\
\text { - GoStop }\end{array}$ & $\begin{array}{c}\text { Dos } 2.620 \text { participantes, } 64 \\
\text { preencheram os critérios para } \\
\text { DI. Desses } 64,8 \text { apresentaram } \\
\text { TDAH }(12,5 \%) .\end{array}$ \\
\hline $\begin{array}{l}\text { Yen JY, Ko CH, Yen CF, Wu HY, Yang MJ. } \\
\text { The comorbid psychiatric symptoms } \\
\text { of Internet addiction: attention deficit } \\
\text { and hyperactivity disorder (ADHD), } \\
\text { depression, social phobia, and hostility. } \\
\text { J Adolesc Health. 2007;41(1):93-8.22 }\end{array}$ & Taiwan & $\begin{array}{l}\text { Estudo } \\
\text { transversal }\end{array}$ & $\begin{array}{l}\text { Determinar a associação } \\
\text { entre DI, depressão, TDAH, } \\
\text { fobia social e hostilidade; } \\
\text { avaliar as diferenças } \\
\text { entre os gêneros na DI e } \\
\text { os sintomas psiquiátricos } \\
\text { acima mencionados entre os } \\
\text { adolescentes. }\end{array}$ & $\begin{array}{l}n=2.114 \\
\text { Idade média: } \\
16,26(0,99) \\
\text { Masc.: } 1.204 \\
\text { Fem.: } 910\end{array}$ & $\begin{array}{c}\text { - CIAS; } \\
\text { - ADHDS; } \\
\text { - DSM-IV TR; } \\
\text { - CES-D; } \\
\text { - SPIN; } \\
\text { - CHI-SF } \\
\text { - Questionário dos } \\
\text { dados demográficos }\end{array}$ & $\begin{array}{l}\text { Adolescentes com DI } \\
\text { apresentaram sintomas mais } \\
\text { elevados de TDAH. }\end{array}$ \\
\hline $\begin{array}{l}\text { Ko CH, Yen JY, Chen CS, Yeh YC, Yen } \\
\text { CF. Predictive values of psychiatric } \\
\text { symptoms for internet addiction in } \\
\text { adolescents: a 2-year prospective } \\
\text { study. Arch Pediatr Adolesc Med. } \\
\text { 2009;163(10):937-43.19}\end{array}$ & Taiwan & $\begin{array}{l}\text { Estudo } \\
\text { transversal } \\
\text { longitudinal }\end{array}$ & $\begin{array}{l}\text { Avaliar os valores preditivos } \\
\text { de sintomas psiquiátricos e } \\
\text { determinar as diferenças de } \\
\text { gênero no valor preditivo } \\
\text { de sintomas psiquiátricos } \\
\text { para a ocorrência da DI em } \\
\text { adolescentes. }\end{array}$ & $\begin{array}{c}\mathrm{n}=2.293 \\
\text { Idade média: } \\
12,36(0,55) \\
\text { Masc.: } 1.179 \\
\text { Fem.: } 1.114\end{array}$ & $\begin{array}{l}\text { - CIAS } \\
\text { - ADHDS }\end{array}$ & $\begin{array}{l}\text { Desordem de hostilidade e } \\
\text { TDAH foram os preditores mais } \\
\text { significativos na Dl em homens } \\
\text { e mulheres adolescentes, } \\
\text { respectivamente. }\end{array}$ \\
\hline $\begin{array}{l}\text { Bozkurt H, Coskun M, Ayaydin H, Adak } \\
\text { I, Zoroglu SS. Prevalence and patterns } \\
\text { of psychiatric disorders in referred } \\
\text { adolescents with Internet addiction. } \\
\text { Psychiatry Clin Neurosci. 2013;67(5):352-9.13 }\end{array}$ & Turquia & $\begin{array}{l}\text { Estudo } \\
\text { transversal }\end{array}$ & $\begin{array}{l}\text { Investigar as características } \\
\text { sociodemográficas e a } \\
\text { prevalência de padrão de } \\
\text { desordens psiquiátricas em } \\
\text { jovens com DI. }\end{array}$ & $\begin{array}{c}\mathrm{n}=60 \\
10-18 \text { anos } \\
\text { Masc.: } 45 \\
\text { Fem.: } 15\end{array}$ & $\begin{array}{l}\text { - YIAS } \\
\text { - K-SADS-PL } \\
\text { - DSM-IV }\end{array}$ & $\begin{array}{l}\text { Todos os indivíduos tinham } \\
\text { uma e } 88,3 \% \text { ( } n=53 \text { ) tinham } \\
\text { pelo menos duas comorbidades } \\
\text { psiquiátricas, sendo o TDAH ( } n \\
\quad=53 \text { ) }=83,3 \% .\end{array}$ \\
\hline $\begin{array}{l}\text { Kaess M, Durkee T, Brunner R, Carli } \\
\text { V, Parzer P, Wasserman C, et al. } \\
\text { Pathological Internet use among } \\
\text { European adolescents: psychopathology } \\
\text { and self-destructive behaviours. } \\
\text { Eur Child Adolesc Psychiatry. } \\
\text { 2014;23(11):1093-102.14 }\end{array}$ & $\begin{array}{l}\text { Alemanha } \\
\text { Suíça }\end{array}$ & $\begin{array}{l}\text { Estudo } \\
\text { transversal }\end{array}$ & $\begin{array}{l}\text { Investigar a associação } \\
\text { entre } 0 \text { uso patológico da } \\
\text { internet (PIU), psicopatologia } \\
\text { e o comportamento } \\
\text { autodestrutivo em } \\
\text { adolescentes escolares em } 11 \\
\text { países europeus. }\end{array}$ & $\begin{array}{c}\mathrm{n}=11.356 \\
\text { Idade média: } \\
14,9(0,88) \\
\text { Masc.: } 4.856 \\
\text { Fem.: } 6.500\end{array}$ & $\begin{array}{l}-T D Q \\
-Y D Q \\
-S D Q\end{array}$ & $\begin{array}{l}\text { Uso patológico da internet: } \\
\qquad 4.2 \%) \\
\text { Uso patológico da internet: } \\
\text { feminino }(3,9 \%) \text { masculino } \\
(4,7 \%) \text { Hiperatividade/ } \\
\text { inatenção }(37,2 \%)\end{array}$ \\
\hline $\begin{array}{l}\text { Yen CF, Chou WJ, Liu TL, Yang P, Hu HF. } \\
\text { The association of Internet addiction } \\
\text { symptoms with anxiety, depression and } \\
\text { self-esteem among adolescents with } \\
\text { attention-deficit/hyperactivity disorder. } \\
\text { Compr Psychiatry. 2014;55(7):1601-8. } .^{5}\end{array}$ & Taiwan & $\begin{array}{c}\text { Estudo } \\
\text { transversal }\end{array}$ & $\begin{array}{l}\text { Examinar as associações } \\
\text { entre DI e ansiedade, } \\
\text { depressão e autoestima em } \\
\text { adolescentes diagnosticados } \\
\text { com TDAH em Taiwan. }\end{array}$ & $\begin{array}{l}\mathrm{n}=287 \\
\text { adolescentes/ } \\
\text { TDAH } \\
\text { Idade média: } \\
\text { 13,1 }(2,0) \\
\text { Masc.: } 251 \\
\text { Fem.: } 36\end{array}$ & $\begin{array}{l}- \text { CIAS } \\
- \text { MASC-T } \\
- \text { CES-D } \\
\text { - RSES } \\
\text { - SNAP-IV } \\
\text { - DSM-IV }\end{array}$ & $\begin{array}{l}\text { 15,7\% dos adolescentes já } \\
\text { diagnosticados com TDAH } \\
\text { apresentaram DI. }\end{array}$ \\
\hline $\begin{array}{l}\text { Chen YL, Chen SH, Gau SS. ADHD } \\
\text { and autistic traits, family function, } \\
\text { parenting style, and social adjustment } \\
\text { for Internet addiction among children } \\
\text { and adolescent Res Dev Disabil. } \\
\text { 2015;39:20-31.23 }\end{array}$ & Taiwan & $\begin{array}{l}\text { Estudo } \\
\text { transversal } \\
\text { longitudinal }\end{array}$ & $\begin{array}{l}\text { Investigar a prevalência, } \\
\text { os preditores e fatores } \\
\text { relacionados a Dl entre } \\
\text { estudantes do } 1^{\circ} \text { e } 2^{\circ} \text { grau de } \\
\text { uma escola em Taiwan. }\end{array}$ & $\begin{array}{l}\mathrm{n}=1.153 \\
\text { Masc.: } 573 \\
\text { Fem.: } 580 \\
\text { Pais }=997\end{array}$ & $\begin{array}{l}\text { - CIAS } \\
\text { - Questionário de } \\
\text { frequência, uso e } \\
\text { tempo na internet } \\
\text { - SNAP-IV } \\
\text { - DSM-IV }\end{array}$ & $\begin{array}{l}\text { A prevalência de DI decresceu } \\
\text { de } 11,4 \% \text { (1a onda) para } 10,6 \% \\
\text { (2a onda). Sintomas elevados } \\
\text { de TDAH têm efeito preditivo na } \\
\text { adição de internet. }\end{array}$ \\
\hline $\begin{array}{l}\text { Chou WJ, Liu TL, Yang P, Yen CF, Hu HF. } \\
\text { Multi-dimensional correlates of Internet } \\
\text { addiction symptoms in adolescents with } \\
\text { attention-deficit/hyperactivity disorder. } \\
\text { Psychiatry Res. 2015;225(1-2):122-8. } .^{16}\end{array}$ & Taiwan & $\begin{array}{c}\text { Estudo } \\
\text { transversal }\end{array}$ & $\begin{array}{l}\text { Examinar a associação entre } \\
\text { a DI com: sensibilidade ao } \\
\text { reforço, fatores familiares, } \\
\text { atividade em internet, entre } \\
\text { adolescentes em Taiwan } \\
\text { com TDAH. }\end{array}$ & $\begin{array}{l}\mathrm{n}=287 \\
\text { adolescestes c/ } \\
\text { TDAH } \\
11-18 \text { anos } \\
\text { Masc.: } 251 \\
\text { Fem.: } 36\end{array}$ & $\begin{array}{l}- \text { CIAS } \\
\text {-SNAP-IV } \\
\text { - DSM-IV } \\
\text { - BIS/BAS }\end{array}$ & $\begin{array}{l}\text { Não se encontrou associação } \\
\text { significante dos sintomas de DI } \\
\text { com os sintomas de TDAH. }\end{array}$ \\
\hline $\begin{array}{l}\text { Metin 0, Saracli 0, Atasoy N, Senormanci } \\
\text { 0, Kardes VC, Acikgoz HO, et al. } \\
\text { Association of internet addiction in } \\
\text { high school students with ADHD and } \\
\text { tobacco/alcohol use. Düşüunen Adam. } \\
\text { 2015;28(3):204." }\end{array}$ & Turquia & $\begin{array}{l}\text { Estudo } \\
\text { transversal }\end{array}$ & $\begin{array}{l}\text { Avaliar a associação entre } \\
\text { Dl e TDAH e tabagismo e } \\
\text { álcool em estudantes do } \\
\text { ensino médio que vivem na } \\
\text { província de Zonguldak. }\end{array}$ & $\begin{array}{l}\mathrm{n}=771 \\
\text { Idade média: } \\
\text { 16,9 }(0,9) \\
\text { Masc.: } 311 \\
\text { Fem.: } 460\end{array}$ & $\begin{array}{l}\text { - CIAS } \\
\text { - Adult ADD/ADHD; } \\
\text { - Formulário de dados } \\
\text { sociodemográficos }\end{array}$ & $\begin{array}{l}\text { Taxa de TDAH era maior entre } \\
\text { aqueles com DI }(36,1 \%) \text { do que } \\
\text { entre aqueles sem DI }(9,6 \%) \text {. A } \\
\text { Dl está associada ao TDAH e ao } \\
\text { consumo de álcool/tabaco. }\end{array}$ \\
\hline $\begin{array}{l}\text { Yılmaz S, Hergüner S, Bilgiç A, Işı Ü. } \\
\text { Internet addiction is related to attention } \\
\text { deficit but not hyperactivity in a sample } \\
\text { of high school students. Int J Psychiatry } \\
\text { Clin Pract. 2015;19(1):18-23.18 }\end{array}$ & Turquia & $\begin{array}{l}\text { Estudo } \\
\text { transversal }\end{array}$ & $\begin{array}{c}\text { Avaliar os efeitos dos } \\
\text { sintomas do TDAH na DI entre } \\
\text { os estudantes do ensino } \\
\text { médio. }\end{array}$ & $\begin{array}{l}n=640 \\
\text { Idade média: } \\
15,96(0,84) \\
\text { Masc.: } 309 \\
\text { Fem.: } 331\end{array}$ & $\begin{array}{c}\text { - Formulário de } \\
\text { Informaçãa Pessoal } \\
\text { - IAS } \\
\text { - CASS-S }\end{array}$ & $\begin{array}{l}\text { Déficit de atenção e jogar jogos } \\
\text { on-line foram preditores } \\
\text { significativos de Dl em ambos os } \\
\text { sexos. A hiperatividade e outros } \\
\text { recursos de uso da internet não } \\
\text { previram DI. }\end{array}$ \\
\hline
\end{tabular}




\begin{tabular}{|c|c|c|c|c|c|c|}
\hline Referência completa & País & $\begin{array}{c}\text { Desenho } \\
\text { de pesquisa }\end{array}$ & Objetivo & Amostra & Instrumentos & Principais resultados \\
\hline $\begin{array}{l}\text { Weinstein A, Yaacov Y, Manning M, } \\
\text { Danon P, Weizman A. Internet Addiction } \\
\text { and Attention Deficit Hyperactivity } \\
\text { Disorder Among Schoolchildren. Isr Med } \\
\text { Assoc J. 2015Dec;17(12):731-4. } .^{21}\end{array}$ & Israel & $\begin{array}{c}\text { Estudo } \\
\text { transversal }\end{array}$ & $\begin{array}{l}\text { Investigar a relação entre uso } \\
\text { problemático da internet e } \\
\text { TDAH entre alunos de uma } \\
\text { escola religiosa judaica. }\end{array}$ & $\begin{array}{c}n=100 \\
\text { 13-15 anos } \\
\text { Masc. sem } \\
\text { TDAH: } 50 \\
\text { Masc. c/ TDAH: } \\
50\end{array}$ & $\begin{array}{l}\text { - IAT } \\
\text { - Questionário sobre } \\
\text { horas na internet e } \\
\text { horário de dormir }\end{array}$ & $\begin{array}{l}\text { Crianças com TDAH tiveram } \\
\text { pontuações mais altas na DI, } \\
\text { utilizaram a internet por mais } \\
\text { horas e foram dormir mais tarde } \\
\text { do que aquelas sem TDAH. }\end{array}$ \\
\hline $\begin{array}{l}\text { Seyrek S, Cop E, Sinir H, Ugurlu M, Şenel } \\
\text { S. Factors associated with Internet } \\
\text { addiction: Cross-sectional study of } \\
\text { Turkish adolescents. Pediatr Int. } \\
\text { 2017;59(2):218-22. }{ }^{20}\end{array}$ & Turquia & $\begin{array}{c}\text { Estudo } \\
\text { transversal }\end{array}$ & $\begin{array}{l}\text { Investigar a prevalência } \\
\text { de DI e a relação } \\
\text { entre características } \\
\text { sociodemográficas, } \\
\text { depressão, ansiedade, } \\
\text { sintomas de TDAH e DI em } \\
\text { adolescentes. }\end{array}$ & $\begin{array}{c}\mathrm{n}=468 \\
12-17 \text { anos } \\
\text { Masc.: } 204 \\
\text { Fem.: } 264\end{array}$ & $\begin{array}{c}\text { - YIAS } \\
\text { - CDI } \\
\text { - BAI } \\
\text { - CPRS } \\
\text { - CTRS } \\
\text { - Hollingshead- } \\
\text { Redlichscale }\end{array}$ & $\begin{array}{l}\text { 1,6\% dos alunos foi identificado } \\
\text { com DI, enquanto 16,2\% } \\
\text { tiveram possível DI. Houve } \\
\text { correlação significativa entre } \\
\text { DI e TDAH. Não foi encontrada } \\
\text { relação estatisticamente } \\
\text { significante entre DI e sexo, } \\
\text { idade, índice de massa } \\
\text { corpórea, escolaridade e status } \\
\text { social. }\end{array}$ \\
\hline
\end{tabular}

Adult ADD/ADHD Diagnostic and Assessment Inventory; Children's Depression Inventory (CDI); Attention-deficit/Hyperactivity Disorder Self-rated Scale (ADHDS); Beck Anxiety Inventory (BAI); Center for Epidemiological Studies Depression Scale (CES-D); Conners-Wells Adolescent Self-Report Scale-Short Form (CASS-S); Conners' Parent Rating Scale (CPRS); Conners' Teacher Rating Scale (CTRS); DSM-IV; Diagnostic Questionnaire for Internet Addiction (YDQ); entrevista clínica estruturada (K-SADS); Barrat Impulsiveness Scale-11 (BIS-11); escala para desordem afetiva e esquizofrenia (K-SADS-PL); GoStop Impulsivity Paradigm (GoStop); Hollingshead-Redlich scale; Internet Addiction Scale (IAS); Chen Internet Addiction Scale (CIAS); Multidimensional Anxiety Scale for Children (MASC-T); Rosenberg Self-Esteem Scale (RSES); DSM-IV TR; Social Phobia Inventory (SPIN); Strengths and Difficulties Questionnaire (SDQ); behavior inhibition system and behavior approach system (BIS/BAS); Center for Epidemiological Studies' Depression Scale (CES-D); Chinese Hostility Inventory-Short Form (CHI-SF); Chinese version of the Swanson, Nolan, and Petham IV (SNAP-IV); Young's Diagnostic Questionnaire (TDQ); Young's Internet Addiction Scale (YIAS) ou Young's Internet Addiction Test (IAT).

Inicialmente os trabalhos foram analisados quanto a características como ano de publicação, veículo em que foram publicados, desenho metodológico dos estudos, origem dos estudos, objetivos e amostra. Posteriormente, foram realizadas leitura e análise crítica dos artigos buscando agrupar os trabalhos em categorias temáticas. As seguintes categorias foram formuladas: Relação entre DI e TDAH, Outros fatores associados à DI, Instrumentos para avaliação de DI e dados de incidência.

Observa-se uma irregularidade na publicação de estudos: períodos sem publicação (por exemplo, 2010 a 2012) ou com intensa concentração (em 2015, por exemplo, foram publicados cinco artigos, $41,6 \%$ do total selecionado). Nove (75\%) dos 12 estudos foram resgatados na base de pesquisa multidisciplinar Scopus, dois exclusivamente na PsycINFO $(16,7 \%)$ e um na Cinahl (8,3\%). Entre os artigos analisados, sete $(58,3 \%)$ foram publicados em revistas da área específica da psiquiatria'12-18, dois (16,7\%), da pediatria ${ }^{19,20}$, um $(8,3 \%)$, da saúde coletiva ${ }^{21}$ e dois (16,7\%) são periódicos de conhecimento multidisciplinar ${ }^{22,23}$. Dez estudos utilizaram desenho metodológico transversal e dois, longitudinais ${ }^{19,23}$, com o objetivo de identificar possíveis preditores da DI.

Em relação à origem dos estudos, observa-se a concentração de produção científica em duas regiões: Ásia (predominantemente Taiwan), com 50\% ${ }^{12,15,16,19,22,23}$ dos artigos selecionados, e Oriente Médio (predominantemente Turquia), com 41,7\%13,17,18,20,21. Um dos estudos é multicêntrico, desenvolvido em colaboração entre pesquisadores de 11 países, tendo como sedes Alemanha e Suíça ${ }^{14}$.

A maioria dos trabalhos desenvolvidos teve como objetivo identificar diversas psicopatologias que poderiam estar associadas à $\mathrm{Dl}$, com exceção de três trabalhos que focalizaram a relação específica entre a DI e o TDAH'12,18,21 e dois artigos que restringiram sua amostra de pesquisa a apenas adolescentes diagnosticados com TDAH ${ }^{15,16}$, com o intuito de identificar a incidência da DI nessa população.

Quanto à classificação de gênero na amostra, um único estudo focalizou sua pesquisa no adolescente masculino ${ }^{21}$; todos os demais trabalhos incluíram participantes masculinos e femininos.

\section{Relação entre DI e TDAH}

Dos 12 artigos selecionados, 11 estudos indicaram haver relação entre DI e sintomas de TDAH, mostrando as possíveis associações entre essas condições ${ }^{12-15,17-23}$, inclusive quando comparados adolescentes com e sem DI 12,17,22.

Estudos indicaram que, entre várias comorbidades associadas à DI, o TDAH é uma das mais prevalentes ${ }^{12,13,17}$. Sintomas de hiperatividade, déficit de atenção e impulsividade, de forma independente, também têm sido encontrados em associação com DI 12,14,18. Algumas investigações apontaram que o TDAH é um preditor para DI19,23 e destacaram a importância de programas de prevenção de DI para esses adolescentes. Ainda, adolescentes diagnosticados com TDAH dispendem mais tempo na internet e apresentam escores mais elevados ou sintomas mais severos associados a DI15,21.

Na China, visando obter informações sobre a relação entre DI e impulsividade em adolescentes, pesquisadores identificaram 2,4\% de estudantes com Dl e, entre eles, $12,5 \%$ apresentavam TDAH e 9,4\%, outras comorbidades psiquiátricas. Nesse mesmo estudo, comparando um grupo com DI sem TDAH ou outras comorbidades e um grupo com uso 
normal de internet e sem transtornos psiquiátricos, observou-se que jovens com Dl exibem índices mais elevados de impulsividade ${ }^{12}$.

Um estudo turco, realizado com objetivo de avaliar a associação entre DI, TDAH, tabagismo e uso de álcool entre estudantes, comparando grupos com e sem Dl, encontrou que a prevalência de TDAH foi maior entre estudantes com DI (36,1\% deles) e identificou uma correlação positiva entre as duas desordens ${ }^{13}$.

Com o objetivo de investigar características sociodemográficas e a prevalência de desordens psiquiátricas em jovens com DI, pesquisadores verificaram que $83,3 \%$ dos participantes tinham TDAH ${ }^{17}$. Além de ter sido realizado com amostra clínica, nesse estudo foram incluídos apenas indivíduos com escores muito elevados para DI na avaliação realizada, o que possivelmente resultou em prevalência maior do que a encontrada em outras investigações $12,14,17,20$.

Ao pesquisar a associação entre DI, psicopatologia e comportamento autodestrutivo, estudantes de 11 países europeus foram avaliados e detectaram-se 4,2\% deles com $\mathrm{DI}$, entre os quais $37,2 \%$ apresentavam sintomas de hiperatividade e/ou inatenção, que se mostraram como preditores para UPI. A associação entre UPI e os sintomas de hiperatividade/inatenção foi mais forte entre as meninas ${ }^{14}$.

Outro estudo, que buscou conhecer características de uso da internet entre estudantes do ensino médio e analisar separadamente os sintomas de déficit de atenção e de hiperatividade em adolescentes turcos, encontrou que o déficit de atenção e a participação em jogos on-line foram preditores significativos de DI, enquanto a hiperatividade e uso de outros recursos de internet, como busca por informação e redes sociais, não foram associados a ela ${ }^{18}$.

Ao avaliar o valor preditivo de sintomas psiquiátricos para a ocorrência de DI, pesquisadores verificaram que, entre mulheres, depressão, TDAH, fobia social e hostilidade foram preditores de DI, enquanto, entre homens, apenas TDAH e hostilidade mostraram valor preditivo. Para ambos, TDAH e hostilidade, nessa ordem, foram os preditores mais significativos de $\mathrm{DI}^{19}$. Outra investigação detectou que, além do TDAH, gênero masculino, estilo parental protetor, desempenho acadêmico baixo e problemas de relacionamento com pares também se mostraram preditores de $\mathrm{Dl}^{23}$.

Verificando as associações da gravidade de sintomas de DI com diferentes dimensões de sintomas ansiosos, depressivos e de autoestima apresentados por adolescentes com TDAH, pesquisadores encontraram que escores de sintomas físicos mais elevados e evitação de danos mais baixos (associados à ansiedade), taxas de desconforto somático elevadas (associadas à depressão) e escores de autoestima mais baixos mostraram associação significativa com sintomas mais severos de $\mathrm{Dl}^{15}$. Outro estudo comparou meninos com e sem TDAH quanto à DI, tempo gasto com a internet e pa- drões de sono e identificou que indivíduos com TDAH têm escores mais elevados para DI, dispendem mais tempo na internet e tendem a dormir após a meia-noite ${ }^{21}$.

Em uma amostra taiwanesa, adolescentes com DI apresentaram sintomas mais elevados para TDAH e depressão, fobia social e hostilidade, e este último só se associou à DI para o sexo masculino ${ }^{22}$.

Por fim, outro estudo taiwanês, realizado com 287 adolescentes com diagnóstico de TDAH, não encontrou associação entre sintomas de DI e sintomas de inatenção, hiperatividade e impulsividade, divergindo das outras investigações. A baixa satisfação com as relações familiares foi o fator preditivo mais forte da DI. Os resultados encontrados indicaram que nem todos os adolescentes que estavam recebendo tratamento para TDAH eram vulneráveis à $\mathrm{DI}^{16}$. Como o grupo de adolescentes participantes da pesquisa estava em tratamento para o TDAH, os autores sugerem que é possível que os sintomas de TDAH tenham melhorado após tratamento clínico, atenuando também os sintomas de Dl.

A maioria dos estudos encontrados teve como objetivo o diagnóstico da DI e a identificação de possíveis psicopatologias associadas, chegando a resultados de índice associativo expressivo com o TDAH. Contudo, não tinham como objetivo o aprofundamento no estudo dos possíveis mecanismos que poderiam explicar essa relação, muito provavelmente pela complexidade do comportamento adicto e da associação com outras comorbidades. Alguns mecanismos biopsicossociais, como a tendência de ficar facilmente entediado, a aversão à recompensa demorada e o déficit de motivação, foram citados para explicar a associação significativa entre DI e TDAH ${ }^{13}$, porém não foram profundamente discutidos.

\section{Outros fatores associados à DI}

Investigações sobre possíveis associações entre DI e TDAH apontaram outros fatores associados à DI, como o maior grau de escolaridade, que apresentou impacto positivo no tempo de uso da internet (mas não na dependência), lembrando que tempo de permanência na internet não é isoladamente um indicador de $\mathrm{Dl}^{23}$. Conflitos familiares, sintomas físicos (dores de cabeça, pescoço, costas, dormência nos dedos, lacrimação nos olhos), baixo desempenho escolar e problemas elevados com os pares ${ }^{13,14,23}$ foram queixas autorrelatadas pelos adolescentes acometidos pela DI. Aspectos relacionados a transtornos no sono também foram observados em adolescentes que apresentaram DI, quando comparados ao grupo controle ${ }^{21}$. Status socioeconômico dos pais e relações familiares menos satisfatórias estavam significativamente associados com sintomas mais severos da Dl, provavelmente por causa de um estilo de vida mais sedentário ${ }^{16}$. Outros preditores são sugeridos para a DI, além do TDAH: baixa satisfação com relações familiares e baixo desempenho acadêmico ${ }^{16,23}$. 
A internet pode ser utilizada de diferentes formas; assim, alguns estudos buscaram conhecer as atividades mais buscadas pelos adolescentes diagnosticados com Dl e encontraram que o maior tempo gasto é com jogos on-line (principalmente entre os adolescentes diagnosticados com TDAH), seguido por bate-papo ou redes sociais e depois por atividades escolares ${ }^{12,23}$.

Observou-se diferença entre a incidência de DI quanto ao sexo em alguns estudos. Adolescentes do sexo masculino apresentaram incidência maior de $\mathrm{Dl}^{13,14,23}$, utilizaram mais tempo com jogos on-line ${ }^{13}$ e apresentaram sintomas mais altos de hostilidade ${ }^{19,22}$ associados à DI. Por outro lado, adolescentes do sexo feminino apresentaram menor incidência de $\mathrm{DI}^{13,14,23}$ e demonstraram maior interesse em redes sociais ${ }^{13}$. Entretanto, um dos trabalhos teve resultado conflitante com os anteriores, não observando diferença significativa na DI quanto à variável sexo ${ }^{20}$. Além disso, levando-se em consideração a variável sexo em adolescentes diagnosticados com $\mathrm{Dl}$, sintomas de depressão foram observados em jovens de ambos os sexos ${ }^{22}$.

\section{Instrumentos para avaliação de DI e dados de incidência}

Foram identificadas cinco nomenclaturas distintas de instrumentos para avaliar e mensurar a DI correspondentes a quatro distintos questionários. Uma característica comum a todos os instrumentos nos 12 artigos é que eles consistem em questionários preenchidos pelos próprios adolescentes, ou seja, de autorrelato. O instrumento mais utilizado foi o Chen Internet Addiction Scale (CIAS) ${ }^{15-17,19,22,23}$, seguido pelo Young's Internet Addiction Scale (YIAS) $)^{13,20}$ ou Young's Internet Addiction Test (IAT) ${ }^{21}$, Young's Diagnostic Questionnaire (YDQ) ${ }^{12,14}$ e Internet Addiction Scale (IAS) ${ }^{18}$.

O CIAS avalia a severidade dos sintomas de DI no último mês, a partir de 26 itens avaliados em uma escala Likert de quatro pontos, com escores que variam de 26 a 104 - valores mais elevados indicam maior nível de gravidade. Escores iguais ou maiores que 64 indicam a presença de D/24,25.

O YIAS, também conhecido por YIAT ${ }^{20}$ ou IAT, avalia em que extensão o uso da internet afeta a rotina diária, vida social, produtividade, padrões de sono e emoções. É composto por 20 questões respondidas em uma escala Likert de cinco pontos (variando de raramente a sempre), e os escores totais podem variar de 20 a 100, com valores mais altos indicando tendência à adição. Os escores permitem discriminar entre três condições: controle sobre o uso de internet (de 20 a 39 pontos), problemas frequentes devidos ao uso de internet (40 a 69 pontos) e problemas significativos causados pela internet (70 pontos ou mais) $)^{13,26}$.

O YDQ ou IADQ, desenvolvido por Young em 1998, consiste de oito questões do tipo sim/não que avaliam atividade on-line, com base em critérios diagnósticos para dependên- cia de substância do DSM-IV. Quando cinco ou mais critérios estão presentes, o usuário é classificado com Di²7.

O IAS consiste em um instrumento com 36 itens, também baseado nos critérios diagnósticos do DSM-IV para dependência de substância. É avaliado em uma escala Likert de cinco pontos, que varia de nunca a sempre, e escores mais altos representam maior $\mathrm{Dl}^{28}$.

Com respeito à incidência de DI, os estudos mostram variação nas informações, inclusive em decorrência do perfil amostral. Um estudo europeu identificou 4,2\% de uso problemático da internet (3,9\% entre mulheres e 4,7\% entre homens) ${ }^{14}$. Outro estudo, chinês, identificou $2,4 \%$ de adolescentes que preencheram os critérios para DI (64 em uma amostra de 2.620 adolescentes) $)^{12}$. Na Turquia, 1,6\% de uma amostra de 468 adolescentes foi detectado como tendo DI e 16,2\% como tendo provável D|20. Entre adolescentes com TDAH, observou-se incidência de 5,7\% de DI ${ }^{15}$. Observou-se grande variabilidade entre os estudos quanto ao delineamento e instrumentos de medida de DI, bem como identificou-se que várias questões referentes ao diagnóstico ainda não estão totalmente esclarecidas.

\section{DISCUSSÃO}

A literatura propõe que a DI seja considerada um novo transtorno psiquiátrico do século XXI ${ }^{1,2}$. Uma das possíveis causas da concentração de estudos dessa área na Ásia e no Oriente Médio talvez seja o reflexo do desenvolvimento econômico desses países, proporcionando um desenvolvimento tecnológico mais acelerado e facilitando o acesso a equipamentos e internet pela população adolescente, com índices na população de estudantes diagnosticados com DI na Ásia variando entre $2,4 \%$ e 10,6\%12,23,29,30. A presença de computador em casa e o fácil acesso residencial à internet são uma realidade presente nos países em que se concentram as pesquisas sobre $\mathrm{Dl}$, os quais possuem maior desenvolvimento econômico; em uma das amostras 100\% dos adolescentes possuem computadores em casa ${ }^{13}$.

A ausência de critérios definidos de diagnóstico², a associação de facilidades e dificuldades que a utilização da internet proporciona no cotidiano e o seu uso excessivo representam comportamentos disfuncionais que têm sido relacionados a fatores comuns, como a impulsividade e fatores específicos (por exemplo: motivos diversificados e cognições disfuncionais que perpetuam esses comportamentos) $)^{31}$, e dificultam o diagnóstico, fazendo com que a DI ainda não esteja presente no DSM-5. A dependência em internet não envolve apenas o uso da internet para acesso a jogos on-line; os critérios de diagnóstico da DI implicam uma utilização mais diversificada como mídias sociais ou outros usos. Nesse sentido, pesquisas futuras sobre o seu uso 
excessivo precisam seguir diretrizes similares e são esses os esforços de pesquisadores na elucidação de quais critérios comportamentais e/ou emocionais devem ser levados em consideração em relação ao uso saudável ou não da internet para a inclusão da Dl como psicopatologia nas próximas edições do DSM.

A utilização da internet cada vez mais faz parte das atividades cotidianas de adolescentes e aos poucos vem ganhando espaço nas escolas, tanto no sentido motivacional quanto de apoio pedagógico no processo de ensino-aprendizagem. Os adolescentes diagnosticados com DI fizeram maior uso da internet para jogos ${ }^{12,23}$ e em um dos estudos foi a atividade mais frequente entre os adolescentes também diagnosticados com TDAH ${ }^{23}$. Os resultados das pesquisas também indicam que os adolescentes com sintomas de hiperatividade e impulsividade demonstraram maior inabilidade em controlar o uso da internet, sendo, então, um fator de risco ao desenvolvimento da $\mathrm{DI}^{12}$. Os trabalhos não apontam o uso educacional como sendo o mais atrativo para os adolescentes com características de $\mathrm{DI}^{12,23}$, mas o fato de a adolescência ser um período em que a atividade escolar é uma atividade significativa no cotidiano. Com o incentivo ao uso da internet, sem monitoramento sobre em quais locais na rede os adolescentes estão efetivamente navegando por mais tempo, pode-se abrir espaços para o uso descontrolado da internet por eles.

Alguns mecanismos podem explicar a coexistência entre TDAH e DI. A resposta rápida e a recompensa imediata fornecida pela internet, principalmente nos jogos on-line, reduziriam a sensação de tédio nos indivíduos com TDAH, os quais facilmente se entediam e possuem aversão pela recompensa atrasada ${ }^{11,32}$. A dopamina liberada durante jogos pode ajudar os jogadores a se manterem concentrados nos jogos e, assim, a terem melhor desempenho ${ }^{33}$.

A adolescência é um período do desenvolvimento em que a impulsividade é uma característica marcante ${ }^{5}$. Adolescentes com TDAH, devido à melhora de seu desempenho quando utilizam a internet, preferencialmente para jogos on-line, podem ter dificuldade de autocontrole, possibilitando a utilização mais descontrolada e, assim, contribuindo para a vulnerabilidade à Dl.

No Brasil, o número de habitantes com acesso à internet atingiu 79,9 milhões em 2011, com crescimento de 8\% em relação ao ano anterior ${ }^{34}$, sendo os adolescentes os que mais a acessam. Mesmo não tendo índices tão elevados de acessibilidade tecnológica quando comparados aos países mais expressivos de produção científica no tema, é inquestionável a existência da DI no Brasil'. Num estudo observacional recente, com delineamento transversal, em adolescentes brasileiros, foi observada incidência de $20,7 \%$ de DI. Verificou-se uma porcentagem maior de adolescentes com DI entre os que estudavam em escolas particulares, cuja mãe trabalhava fora da residência e que priorizavam jogos e redes sociais, quando comparados com aqueles cuja atividade principal era referente a e-mails, sites ou outra, não havendo diferença significativa em relação ao sexo nesse aspecto? ${ }^{7}$.

Sintomas elevados de TDAH em adolescentes estão associados à DI e mais associados ao gênero masculino. Alguns estudos longitudinais consideram também que o TDAH pode ser um preditor para a DI, devido ao maior tempo gasto com jogos do que com outras tarefas. Assim, o uso do computador e internet deveria ser monitorado de forma mais criteriosa nessa população.

Nessa revisão, o instrumento mais citado na avaliação da DI foi o CIAS, que não possui validação para o contexto brasileiro. Já o IAT, que é o segundo mais utilizado, possui versão traduzida para o português e encontra-se adaptado para a população-alvo brasileira desde $2012^{35}$. Apesar de apresentar consistência interna satisfatória, ainda não foram realizadas análises de equivalência de mensuração e reprodutibilidade. Assim, sugere-se maior investimento na validação de instrumentos para avaliação da DI no contexto brasileiro ${ }^{35,36}$.

\section{CONCLUSÕES}

Há evidências de associação importante entre DI e TDAH. Contudo, há necessidade de pesquisas que avancem para uma melhor compreensão de como se estabelece a associação entre os dois transtornos, levando em consideração inclusive as atuais divergências em relação à nomenclatura e ao diagnóstico da DI. Nesse sentido, pesquisas que contribuam para a melhor compreensão da DI, de modo a estabelecer estratégias na prevenção e intervenções terapêuticas, também se tornam essenciais, considerando-se que, com o avanço tecnológico, a exposição e uso da internet tendem a aumentar.

\section{CONTRIBUIÇÕES INDIVIDUAIS}

Helena Cristina Medeiros Vieira Schmidek - Contribuiu com a elaboração do artigo, busca bibliográfica, organização dos dados, análise e interpretação destes, e foi uma das responsáveis pela concepção deste artigo e pela aprovação da sua versão final.

Julia Corrêa Gomes - Contribuiu com a elaboração do artigo, busca bibliográfica, organização dos dados, análise e interpretação destes, e foi uma das responsáveis pela concepção deste artigo e pela aprovação da sua versão final.

Patricia Leila dos Santos - Contribuiu com a concepção, desenho, elaboração, revisão crítica do artigo e aprovação da sua versão final.

Ana Maria Pimenta de Carvalho - Contribuiu com a concepção, desenho, revisão crítica do artigo e aprovação da sua versão final. 
Luiz Jorge Pedrão - Contribuiu com a revisão crítica do artigo e aprovação da sua versão final.

Clarissa Mendonça Corradi-Webster - Contribuiu com a revisão crítica do artigo e aprovação da sua versão final.

\section{CONFLITOS DE INTERESSE}

Os autores declaram não haver conflito de interesse e nenhum outro suporte financeiro a ser declarado.

\section{AGRADECIMENTOS}

Os autores agradecem aos profissionais do Serviço de Revisão Bibliográfica da Biblioteca Central de Ribeirão Preto da Universidade de São Paulo.

\section{REFERÊNCIAS}

1. Abreu CN, Karam RG, Góes DS, Spritzer DT. [Internet and videogame addiction: a review]. Rev Bras Psiquiatr. 2008;30(2):156-67.

2. Young KS. Internet addiction: a new clinical phenomenon and its consequences. Am Behav Sci. 2004;48(4):402-15.

3. Brasil. Lei no 8.069, de 13 de julho de 1990. Estatuto da Criança e do Adolescente. Braślia: Ministério da Justiça; 1990.

4. Jorgenson AG, Hsiao RC, Yen CF. Internet addiction and other behavioral addictions. Child Adolesc Psychiatr Clin N Am. 2016;25(3):509-20.

5. van Rooij AJ, Schoenmakers TM, van de Eijnden RJ, van de Mheen D. Compulsive Internet use: the role of online gaming and other internet applications. J Adolesc Health. 2010;47(1):51-7.

6. Ha JH, Yoo HJ, Cho IH, Chin B, Shin D, Kim JH. Psychiatric comorbidity assessed in Korean children and adolescents who screen positive for Internet addiction. J Clin Psychiatry. 2006;67(5):821-6

7. Terroso LB, Argimon IIL. Dependência de internet e habilidades sociais em adolescentes. Estud Pesqu Psicol. 2016;16(1):200-19.

8. Yoo HJ, Cho SC, Ha J, Yune SK, Kim SJ, Hwang J, et al. Attention deficit hyperactivity symptoms and internet addiction. Psychiatry Clin Neurosci. 2004;58(5):487-94.

9. Polanczyk G, de Lima MS, Horta BL, Biederman J, Rohde LA. The worldwide prevalence of ADHD: a systematic review and metaregression analysis. Am J Psychiatry. 2007;164(6):942-8.

10. Rohde LA, Dorneles BV, Costa AC. Intervenções escolares no transtorno de déficit de atenção/hiperatividade. In: Rotta NT, Ohlweilwe L, Riesgo RS, editores. Transtornos da aprendizagem: abordagem neurobiológica e multidisciplinar. Porto Alegre, RS: Artmed; 2006. p. 365-74.

11. $\mathrm{K}_{0} \mathrm{CH}$, Yen JY, Yen $\mathrm{CF}_{\text {, }} \mathrm{Chen} \mathrm{CS}$, Chen CC. The association between Internet addiction and psychiatric disorder: a review of the literature. Eur Psychiatry. 2012;27(1):1-8.

12. Cao F, Su L, Liu T, Gao X. The relationship between impulsivity and Internet addiction in a sample of Chinese adolescents. Eur Psychiatry. 2007;22(7):466-71.

13. Bozkurt H, Coskun M, Ayaydin H, Adak I, Zoroglu SS. Prevalence and patterns of psychiatric disorders in referred adolescents with Internet addiction. Psychiatry Clin Neurosci. 2013;67(5):352-9.
14. Kaess M, Durkee T, Brunner R, Carli V, Parzer P, Wasserman C, et al. Pathological Internet use among European adolescents: psychopathology and self-destructive behaviours. Eur Child Adolesc Psychiatry. 2014;23(11):1093-102.

15. Yen CF, Chou WJ, Liu TL, Yang P, Hu HF. The association of Internet addiction symptoms with anxiety, depression and self-esteem among adolescents with attention-deficit/hyperactivity disorder. Compr Psychiatry. 2014;55(7):1601-8.

16. Chou WJ, Liu TL, Yang P, Yen CF, Hu HF. Multi-dimensional correlates of Internet addiction symptoms in adolescents with attention-deficit/hyperactivity disorder. Psychiatry Res. 2015;225(1-2):122-8

17. Metin 0, Saracli 0, Atasoy N, Senormanci 0, Kardes VC, Acikgoz HO, et al. Association of internet addiction in high school students with ADHD and tobacco/alcohol use. Düşünen Adam. 2015;28(3):204.

18. Y.lmaz $S$, Hergüner $S$, Bilgiç $A$, Işı Ü. Internet addiction is related to attention deficit but not hyperactivity in a sample of high school students. Int J Psychiatry Clin Pract. 2015;19(1):18-23.

19. Ko CH, Yen JY, Chen CS, Yeh YC, Yen CF. Predictive values of psychiatric symptoms for internet addiction in adolescents: a 2-year prospective study. Arch Pediatr Adolesc Med. 2009;163(10):937-43.

20. Seyrek S, Cop E, Sinir H, Ugurlu M, Senel S. Factors associated with Internet addiction: Cross-sectional study of Turkish adolescents. Pediatr Int. 2017;59(2):218-22.

21. Weinstein A, Yaacov Y, Manning M, Danon P, Weizman A. Internet addiction and attention deficit hyperactivity disorder among schoolchildren. Isr Med Assoc J. 2015;17(12):731-4.

22. Yen JY, Ko CH, Yen CF, Wu HY, Yang MJ. The comorbid psychiatric symptoms of Internet addiction: attention deficit and hyperactivity disorder (ADHD), depression, social phobia, and hostility. J Adolesc Health. 2007;41(1):93-8.

23. Chen YL, Chen SH, Gau SS. ADHD and autistic traits, family function, parenting style, and social adjustment for Internet addiction among children and adolescent Res Dev Disabil. 2015;39:20-31.

24. Chen SH, Weng LJ, Su YJ, Wu HM, Yang PF. Development of Chinese Internet Addiction Scale and its psychometric study. Chin J Psychol. 2003;45(3):279-94.

25. Ko CH, Yen JY, Yen CF, Chen CC, Yen CN, Chen SH. Screening for Internet addiction: an empirical study on cut-off points for the Chen Internet Addiction Scale. Kaohsiung J Med Sci. 2005;21(12):545-51.

26. Frangos CC, Frangos CC, Sotiropoulos I.A A Meta-analysis of the reliabilty of Young's Internet Addiction Test. In: Proceedings of the World Congress on Engineering 2012 Vol I - WCE 2012, July 4 - 6, 2012, London, U.K.

27. Johansson A, Götestam KG. Internet addiction: characteristics of a questionnaire and prevalence in Norwegian youth (12-18 years). Scand J Psychol. 2004;45(3):223-9.

28. Nichols LA, Nicki R. Development of a psychometrically sound internet addiction scale: a preliminary step. Psychol Addict Behav. 2004;18(4):381-4.

29. Chou C, Hsiao MC. Internet addiction, usage, gratification, and pleasure experience: the Taiwan college students' case. Computers Educ. 2000;35(1):65-80.

30. Wu HR, Zhu KJ. Path analysis on related factors causing Internet addiction disorder in college students. Chin J Public Health. 2004;20(11):1363-4.

31. Griffiths MD, Kuss DJ, Billieux J, Pontes HM. The evolution of Internet addiction: A global perspective. Addict Behav. 2016;53:193-5.

32. Castellanos FX, Tannock R. Neuroscience of attention-deficit/hyperactivity disorder: the search for endophenotypes. Nat Rev Neurosci. 2002;3(8):617-28.

33. Koepp MJ, Gunn RN, Lawrence AD, Cunningham VJ, Dagher A, Jones T, et al. Evidence for striatal dopamine release during a video game. Nature. 1998;393(6682):266-8.

34. IBOPE. Mais publicidade na rede. Disponível em: http://www.ibope.com.br/ptbr/noticias/ Paginas/Mais\%20publicidade\%20na\%20rede.aspx 2012.

35. Conti MA, Jardim AP, Hearst N, Cordás TA, Tavares H, Abreu CN. Avaliação da equivalência semântica e consistência interna de uma versão em português do Internet Addiction Test (IAT). Rev Psiquiatr Clín. 2012;39(3):106-10.

36. Ngai SS. Exploring the validity of the Internet Addiction Test for students in grades 5-9 in Hong Kong. Int J Adolesc Youth. 2007;13(3):221-37. 